\title{
Competing effects of surface phonon softening and quantum size effects on the superconducting properties of nanostructured $\mathbf{P b}$
}

\author{
Sangita Bose, ${ }^{1 * \#}$ Charudatta Galande, S. P. Chockalingam, Rajarshi Banerjee, ${ }^{2}$ Pratap \\ Raychaudhuri, ${ }^{\dagger 1}$ and Pushan Ayyub ${ }^{\ddagger 1}$ \\ ${ }^{1}$ Department of Condensed Matter Physics and Material Science, Tata Institute of \\ Fundamental Research, Mumbai 400005, India \\ ${ }^{2}$ Department of Materials Science and Engineering, University of North Texas, Denton, \\ Texas 76203-5310, U.S.A.
}

\begin{abstract}
The superconducting transition temperature $\left(T_{C}\right)$ in nanostructured $\mathrm{Pb}$ remains nearly constant as the particle size is reduced from 65 to $7 \mathrm{~nm}$, below which size the superconductivity is lost rather abruptly. In contrast, there is a large enhancement in the upper critical field $\left(H_{C 2}\right)$ in the same size regime. We explore the origin of the unusual robustness of the $T_{C}$ over such a large particle size range in nanostructured $\mathrm{Pb}$, by measuring the temperature dependence of the superconducting energy gap in planar tunnel junctions of $\mathrm{Al} / \mathrm{Al}_{2} \mathrm{O}_{3} /$ nano- $\mathrm{Pb}$. We show that below $22 \mathrm{~nm}$, the electron phonon coupling strength increases monotonically with decreasing particle size, and almost exactly compensates for the quantum size effect, which is expected to suppress $T_{C}$.

\footnotetext{
\# Present address: Max Planck Institute for Solid state Research, Nanoscale Science Department, Stuttgart, Germany.

*Electronic Mail: Sangita.Bose@fkf.mpg.de

† Electronic Mail: pratap@tifr.res.in

‡Electronic Mail: pushan@tifr.res.in
} 
Superconductivity at reduced length scales has been a subject of intense research over the past few decades. ${ }^{1,2,3,4,5,6,7,8,9,10,11}$ Though one may expect changes in the superconducting properties as the system size is reduced below the fundamental length scales such as the coherence length, $\xi(T)$, and the penetration depth, $\lambda_{L}(T)$, it is now established that there is actually a third length scale that finally defines a zero dimensional superconductor. This is the critical particle diameter $\left(D_{C}\right)$ at which the energy level spacing $(\delta)$ arising from the discretization of the energy bands (the "Kubo" gap) equals the superconducting energy gap $(\Delta(0))$. Superconductivity is completely destabilized below this length scale. The existence of such an 'Anderson criterion"2 has been successfully demonstrated in many elemental superconductors such as $\mathrm{Al}^{3}{ }^{3} \mathrm{Sn},{ }^{5} \mathrm{In},{ }^{12} \mathrm{~Pb},{ }^{6,7}$ and $\mathrm{Nb} .{ }^{13}$ However, as the size of the superconductor approaches $D_{C}$, the behavior of the superconducting transition temperature $\left(T_{C}\right)$ is quite different in different systems: superconductors with a weak electron phonon coupling (In, $\mathrm{Al}$, and $\mathrm{Sn}$ ) show an increase in $T_{C}$; the intermediate coupling superconductor $\mathrm{Nb}$ shows a gradual, monotonic decrease in $T_{C}$; while the $T_{C}$ in the strong coupling superconductor, $\mathrm{Pb}$, shows almost no change.

Two competing mechanisms control the $T_{C}$ in nanostructured superconductors. The first arises from the increase in surface to volume ratio with decreasing size. As the surface atoms have a smaller coordination number than the bulk atoms, surface phonons are softer than bulk phonons. This leads to an overall decrease in the phonon frequencies in nanoparticles, ${ }^{14}$ resulting in an enhanced electron-phonon coupling strength ${ }^{15}$ and a higher $T_{C}$. Experimentally, an increase in the electron-phonon coupling can be detected by measuring the dimensionless quantity: $2 \Delta(0) / k_{B} T_{C}$, which monotonically increases with coupling 
strength from its value of 3.52 in the weak coupling limit. This effect could be counteracted by the quantum size effect (QSE) arising from the discretization of the electronic energy bands in small particles and leading to a decrease in the effective density of states, $N(0)$, at the Fermi level. ${ }^{16,17}$ However, within the Bardeen-Cooper-Shrieffer theory, both $\Delta(0)$ and $T_{C}$ are related to $N(0) V$ by the same functional form ( $V$ is the effective electron phonon interaction potential). QSEs, therefore, do not lead to a change in $2 \Delta(0) / k_{B} T_{C}$ with reduction in particle size. To distinguish between the effects of these two mechanisms in nanostructured superconductors, it is necessary to make independent measurements of $T_{C}$ and $\Delta(0)$ as a function of particle size.

We have earlier shown ${ }^{13}$ that in nanocrystalline thin films of $\mathrm{Nb}$, QSEs become apparent below $\approx 20 \mathrm{~nm}$. The QSE-induced reduction in the density of states at the Fermi level decreases $T_{C}$ to almost $50 \%$ of its bulk value as the particle size is reduced from 20 to $8 \mathrm{~nm}$. In the strong coupling superconductor $\mathrm{Pb}$, the bulk superconducting energy gap $(\Delta(0) \approx 1.38 \mathrm{meV})$ as well as the critical size at which superconductivity gets destroyed $\left(D_{C} \approx 6 \mathrm{~nm}\right)$ are close to the corresponding values in $\mathrm{Nb}$. One may therefore expect QSE to play similar roles in $\mathrm{Pb}$ and $\mathrm{Nb}$. The size dependence of $T_{C}$ in $\mathrm{Pb}$ is, however, qualitatively different from that in $\mathrm{Nb}$, decreasing by only by $\approx 13 \%$ as particle size is reduced $^{6,7}$ from bulk to $7 \mathrm{~nm}$, below which it becomes non-superconducting. To understand the robustness of the $T_{C}$ with decreasing size in $\mathrm{Pb}$, we carried out simultaneous measurements of $\Delta(0)$ and $T_{C}$ in planar tunnel junctions consisting of $\mathrm{Al}, \mathrm{Al}_{2} \mathrm{O}_{3}$, and nanostructured $\mathrm{Pb}$ films (with different values of $D$ ), grown by high pressure magnetron sputter deposition. Interestingly, $\Delta(0)$ was found to increase with decreasing size (for $D<20 \mathrm{~nm}$ ) though $T_{C}$ remained 
virtually constant. A measurement of the temperature variation of the gap further indicates a size dependent deviation from the weak coupling BCS behavior in nanostructured $\mathrm{Pb}$, implying an enhancement of the electron phonon coupling strength. Our results suggest that in nano- $\mathrm{Pb}$, the decrease in $T_{C}$ due to quantum size effect is almost exactly offset by the increase in electron phonon coupling strength, down to the Anderson limit.

Nanocrystalline films of $\mathrm{Pb}(\approx 200 \mathrm{~nm}$ thick $)$ were deposited on glass substrates by highpressure magnetron sputtering from elemental $\mathrm{Pb}$ targets (Kurt and Lesker, 99.999\%). The particle size was varied in the range 5-60nm by controlling the sputtering gas (Ar) pressure, the applied power and the deposition time. To prevent oxidation, the nanocrystalline $\mathrm{Pb}$ films were capped with a 40nm thick overlayer of Si grown in-situ using RF-supttering. The mean particle size $(D)$ and size distribution were determined from x-ray diffraction (XRD) line profile analysis using WINFIT software and transmission electron microscopy (TEM). The particle sizes measured by the two methods matched closely. The particle size distribution in each film was approximately $\pm 15 \%$. Figure 1(a) and 1(b) show the bright field TEM patterns obtained from samples with $D_{X R D}=60$ and $14 \mathrm{~nm}$, respectively, the insets showing the corresponding selected area diffraction patterns. The high resolution TEM image of the $D_{X R D}=5 \mathrm{~nm}$ sample (Fig. 1(c)) shows crystalline $\mathrm{Pb}$ grains of $\approx 5 \mathrm{~nm}$ diameter (darker contrast) separated by a disordered intergranular region, possibly consisting of an amorphous $\mathrm{Pb}-\mathrm{O}$ phase. ${ }^{18}$ The $\mathrm{Pb}$ grains are electronically isolated to some degree by the intergranular region. Magnetization and transport measurements were carried out down to 2.2 K using a planar coil ac susceptometer and a magneto-transport set up, respectively. The superconducting energy gap $(\Delta)$ was measured by fabricating planar tunnel junctions of 
$\mathrm{Al} / \mathrm{Al}_{2} \mathrm{O}_{3} /$ nano- $\mathrm{Pb}$ films by a standard method. ${ }^{19} 1 \mathrm{~mm} \mathrm{Al}$ strips were thermally evaporated through a mask on a glass side (thoroughly cleaned in boiling acetone and vapor cleaned in trichloroethylene and propanol). The $\mathrm{Al}$ film was oxidized by exposing to air for 20 minutes. Nanostructured $\mathrm{Pb}$ films were then sputter deposited as cross strips on the $\mathrm{Al}_{2} \mathrm{O}_{3}$ layer using proper masks to produce on each device two $\mathrm{Al} / \mathrm{Al}_{2} \mathrm{O}_{3} /$ nano- $\mathrm{Pb}$ tunnel junctions with effective junction areas of $1 \times 1 \mathrm{~mm}^{2}$. For the tunnel junction with largest particle size $\left(\mathrm{D}_{\mathrm{XRD}}=64 \mathrm{~nm}\right)$ the $\mathrm{Pb}$ strip was thermally evaporated at high vacuum.

Figures 2(a) and (b) show the temperature dependence of the ac magnetic susceptibility and the $\mathrm{dc}$ resistivity for the nano- $\mathrm{Pb}$ films with different particle size. Using commonly accepted criteria, $T_{C}$ was estimated (i) from transport measurements as the temperature at which the resistance dropped to $10 \%$ of its normal state value, and (ii) from ac susceptibility measurements as the temperature at which the real part of susceptibility deviated from zero. The $T_{C}$ obtained from the two methods matched almost exactly (Fig. 3(a)). We observe that the $T_{C}$ does not deviate from the bulk value $(7.24 \mathrm{~K})$ down to $D \approx 14 \mathrm{~nm}$, and decreases by only $\approx 13 \%$ between 14 and 7nm (Fig. 3(a)). Below 7nm, Pb loses superconductivity. The calculated critical particle diameter (Anderson limit) is $6 \mathrm{~nm}^{2}$ This result agrees with the critical size reported by other groups. ${ }^{6,7}$ Fig. 3(a) also brings out the qualitative difference in the size dependence of $T_{C}$ in nano- $\mathrm{Pb}$ and nano- $\mathrm{Nb}$ (data from Ref. 13). In the intermediate coupling superconductor, $\mathrm{Nb}$, a gradual depression of $T_{C}$ starts at comparatively large sizes (above $20 \mathrm{~nm}$ ), and the $T_{C}$ decreases by about $50 \%$ down to $8 \mathrm{~nm}$, below which it becomes non-superconducting. Figure 3(b) shows the upper critical field measured at $4.2 \mathrm{~K}$. The $H_{C 2}$ measured at $4.2 \mathrm{~K}$, shows a monotonic increase with decreasing size down to $7 \mathrm{~nm}$, consistent 
with previous results ${ }^{7}$. In nano-Nb, $H_{C 2}$ shows a non-monotonic size dependence ${ }^{20}$, with an increase down to $20 \mathrm{~nm}$ and steadily decreasing at lower sizes.

The evolution of the superconducting gap $(\Delta)$ with both size and temperature was obtained from 4-probe $I-V$ measurements down to $2.2 \mathrm{~K}$, in planar tunnel junctions of $\mathrm{Al} / \mathrm{Al}_{2} \mathrm{O}_{3} /$ nano$\mathrm{Pb}$. Figures $4(\mathrm{a})$ and (b) show plots of the differential conductance, $G(V)=\mathrm{d} I / \mathrm{d} V$, versus voltage for the samples with $D_{X R D} \approx 64 \mathrm{~nm}$ and $11 \mathrm{~nm}$, respectively. The data was normalized with respect to the conductance values $\left(\mathrm{G}_{\mathrm{n}}\right)$ between $3 \mathrm{mV}$ and $4 \mathrm{mV}$ to avoid the phonon contribution that occur at higher bias. The tunneling spectra ( $G$ vs. $V$ ) were fitted to a theoretical model for tunneling between a superconductor and a normal metal. The $\pm 15 \%$ distribution in particle size leads to a corresponding distribution in $\Delta$, which is accounted for by broadening the BCS density of states ${ }^{21}$ with a linewidth $\Gamma$. The broadened density of states is given by: ${ }^{22} N(E, \Gamma)=\operatorname{Re}\left(\frac{E+i \Gamma}{\sqrt{(E+i \Gamma)^{2}-\Delta^{2}}}\right)$. Thus, there are two fitting parameters, $\Delta$ and $\Gamma$, for each temperature. The gap, obtained by fitting the tunneling spectra, increases from its bulk value ${ }^{23}, \Delta(0) \approx 1.38 \mathrm{meV}$ for the film with $D_{X R D}=64 \mathrm{~nm}$ to $\Delta(0) \approx 1.88 \mathrm{meV}$ for $D_{X R D}=9 \mathrm{~nm}\left(T_{C}=6.9 \mathrm{~K}\right)$. Correspondingly, the coupling strength $2 \Delta(0) / k_{B} T_{C}$ increases from 4.37 for the $64 \mathrm{~nm}$ sample to 6.28 at $9 \mathrm{~nm}$ (Fig. 4(c)). In contrast, in nanostructured $\mathrm{Nb}$, $2 \Delta(0) / k_{B} T_{C}$ remains close to the BCS value ${ }^{13}$ in the same size range. The size-dependent increase in the coupling strength can be independently inferred from the temperature variation of the superconducting gap (Fig. 4(d)). Since bulk $\mathrm{Pb}$ is a strong coupling superconductor, the normalized value of $\Delta$ deviates slightly from the weak coupling BCS curve. However, this deviation progressively increases towards the strong coupling limit as 
the particle size is reduced. Thus, $\mathrm{Pb}$ becomes a stronger coupling superconductor in the nanocrystalline state.

We can also explain the monotonic increase in $H_{C 2}$ down to $7 \mathrm{~nm}$ within the above scenario. The upper critical field is given by: $H_{C 2}=\frac{\phi_{0}}{2 \pi\left(\xi_{0} l_{e f f}\right)^{2}}$, where $\phi_{0}$ is the flux quantum, $\xi_{0}\left[\propto 1 / T_{c} N(0)\right]$ is the Pippard coherence length and $l_{\text {eff }}$, the electronic mean free path. The observed increase in $H_{C 2}$ with size reduction indicates that the decrease in the effective mean free path due to higher fraction of grain boundaries in the nanostructured system, overrides the effect of the decrease in the product $\left[T_{C} N(0)\right]$ that would arise from QSE.

The increase in the electron-phonon coupling strength with decreasing size, inferred from the temperature dependence of the superconducting gap, implies that phonon softening plays an important role in influencing $T_{C}$ in this strong coupling superconductor. The weak coupling BCS equations for $T_{C}$ are thus no longer valid in the nanostructured system. In the strong coupling limit, $T_{C}$ is given by McMillan's equation: ${ }^{15} T_{C}=\frac{\Theta_{D}}{1.45} \exp \left[\frac{-1.04(1+\lambda)}{\lambda-\mu^{*}(1+0.62 \lambda)}\right]$, where $\Theta_{D}$ is the Debye temperature and $\mu^{*}$ is the effective electron-electron repulsion term. McMillan also showed that the $\lambda$ is approximately proportional to the inverse of the average squared phonon frequency: $\left\langle\omega^{2}\right\rangle_{\mathrm{M}}=\langle\omega\rangle /\langle 1 / \omega\rangle$. Thus, a reduction in $\left\langle\omega^{2}\right\rangle_{\mathrm{M}}$, should lead to an increase in $\lambda$. It is clear from McMillan's equation that a higher coupling strength (due to size reduction) should produce higher $T_{C}$. In fact, the enhanced $T_{C}$ observed in nanostructured, weak coupling superconductors such as $\mathrm{Al}, \mathrm{Sn}, \mathrm{Ga}$, and $\mathrm{In}$ are ascribed to 
phonon softening due to surface effects. Why do we not observe a similar increase in $T_{C}$ in the strong coupling superconductor $\mathrm{Pb}$ ? A plausible explanation for the observed size independence of $T_{C}$ is the cancellation of the opposing influences of phonon softening and QSE. The quantization of the electronic wave vector arising from the discretization of the energy levels at small sizes cannot be neglected since it leads to the Anderson criterion that correctly predicts the observed destabilization of superconductivity at $6 \mathrm{~nm}$ in $\mathrm{Pb}$. Also, from our measurements of $T_{C}$ and $\Delta$ in nanostructured $\mathrm{Nb}$, we know that the QSE plays a dominant role ${ }^{13}$ in influencing $T_{C}$ by decreasing the density of states at the Fermi level in small particles. We therefore believe that phonon softening effects (that tend to increase $T_{C}$ ) are almost exactly offset by QSE (that tend to decrease $T_{C}$ ) in $\mathrm{Pb}$ down to $14 \mathrm{~nm}$, resulting in a size invariant $T_{C}$. Between 14 and $7 \mathrm{~nm}$, QSE dominates and produces a $13 \%$ decrease in $T_{C}$. Below $7 \mathrm{~nm}, \mathrm{~Pb}$ is no longer superconducting.

In summary, we have reported a detailed investigation of the evolution of superconductivity with particle size in nanostructured films of $\mathrm{Pb}$. Measurements of the superconducting energy gap and the critical fields in nanostructured $\mathrm{Pb}$ indicate a deviation from the weak coupling BCS behavior. This is indicative of an increase in the electron-phonon coupling strength with reduction in particle size in nano- $\mathrm{Pb}$. The expected increase in $T_{C}$ due to this effect is however not observed, being almost exactly offset by the quantum size effect. Below 7nm, quantum size effects dominate and superconductivity is destroyed, consistent with the Anderson criterion. Our studies provide a natural explanation for the robustness of the superconducting transition temperature in nanosized $\mathrm{Pb}$ particles down to the theoretical limit at which superconductivity is destabilized. 
Acknowledgement: We thank J. John, N. Kulkarni and V. Bagwe for technical assistance. 


\section{Figure Captions}

Figure 1. Bright field TEM images of the nanostructured $\mathrm{Pb}$ films with particle size (a) $D_{X R D}$ $=60 \mathrm{~nm}$ and (b) $D_{X R D}=14 \mathrm{~nm}$, the corresponding selected area diffraction patterns being shown in the insets. (c) High resolution TEM image of the film with $D_{X R D}=5 \mathrm{~nm}$ showing $\mathrm{Pb}$ grains (marked by circles) separated by a disordered intergranular region.

Figure 2. Temperature dependence of the (a) normalized ac susceptibility (b) dc electrical resistance of the nanostructured $\mathrm{Pb}$ films with different particle sizes.

Figure 3. (a) Particle size $(D)$ dependence of the superconducting transition temperature $\left(T_{C}\right)$ of $\mathrm{Pb}$ obtained from electrical transport (circles) and magnetic susceptibility (triangles). The size dependence of $T_{C}$ for $\mathrm{Nb}$ (diamonds) (from ref. 13) is shown for comparison (squares). (b) Variation of the upper critical field $\left(H_{C 2}\right)$ with particle size obtained from magnetoresistance data.

Figure 4. Tunnelling spectra (normalized differential conductance vs. applied voltage) recorded at different temperatures for the nanostructured $\mathrm{Pb}$ films with (a) $D_{X R D}=64 \mathrm{~nm}$, and (b) $D_{X R D}=11 \mathrm{~nm}$. The symbols denote the experimental points and the solid lines are the theoretical fits. (c) Size dependence of the coupling strength, $2 \Delta(0) / k_{B} T_{C}$, for $\mathrm{Pb}$ and $\mathrm{Nb}$ (Ref. 13). (d) Variation of the normalized superconducting energy gap with reduced temperature for $\mathrm{Pb}$ films with $D_{X R D}=64 \mathrm{~nm}, 22 \mathrm{~nm}$ and $11 \mathrm{~nm}$. The solid line is the temperature variation of the gap obtained from the weak coupling BCS equation; (inset) an 
expanded portion of the same data showing more clearly the increasing deviation from weak coupling behaviour close to $\mathrm{T}_{\mathrm{c}}$. 

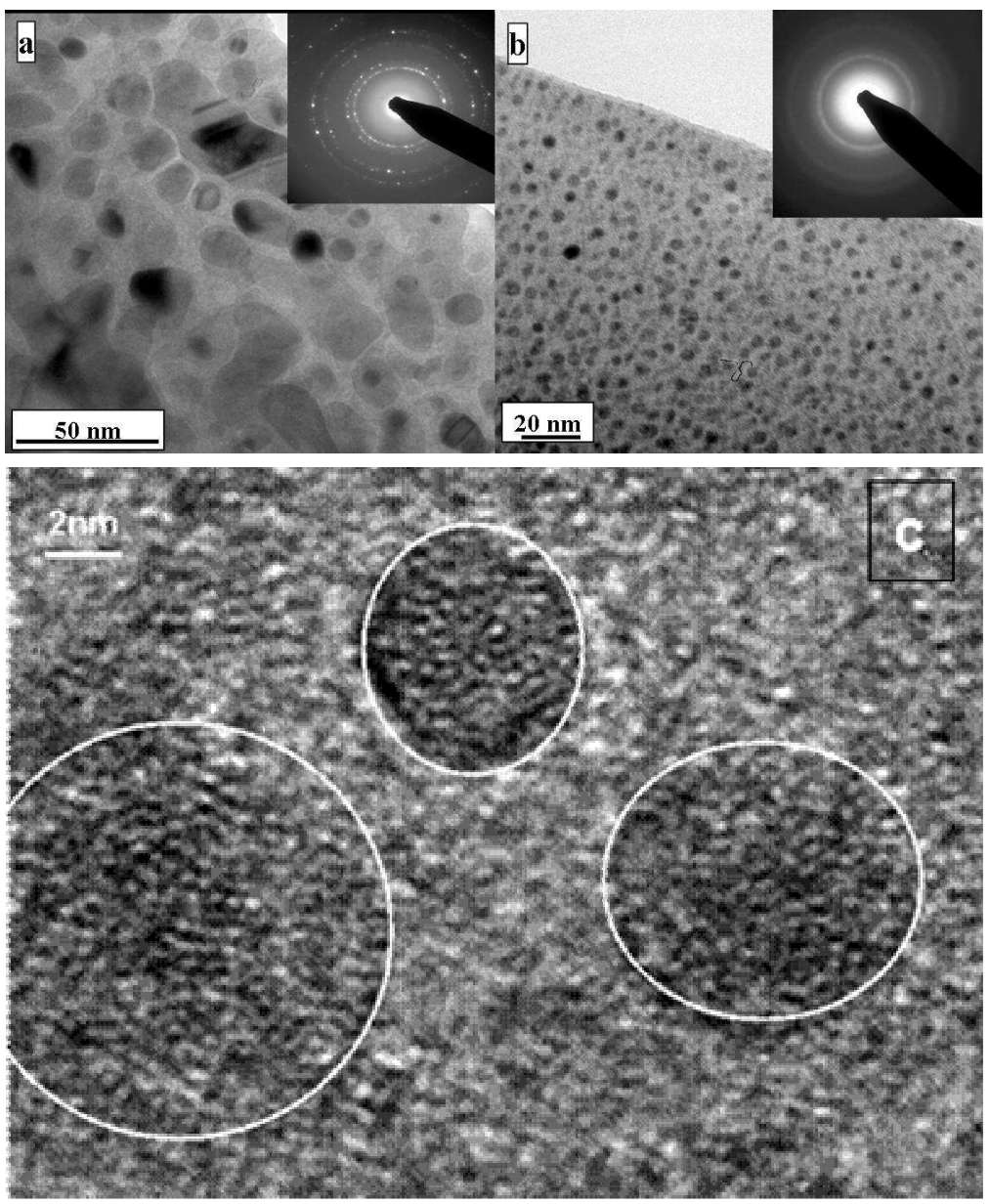

Figure 1 


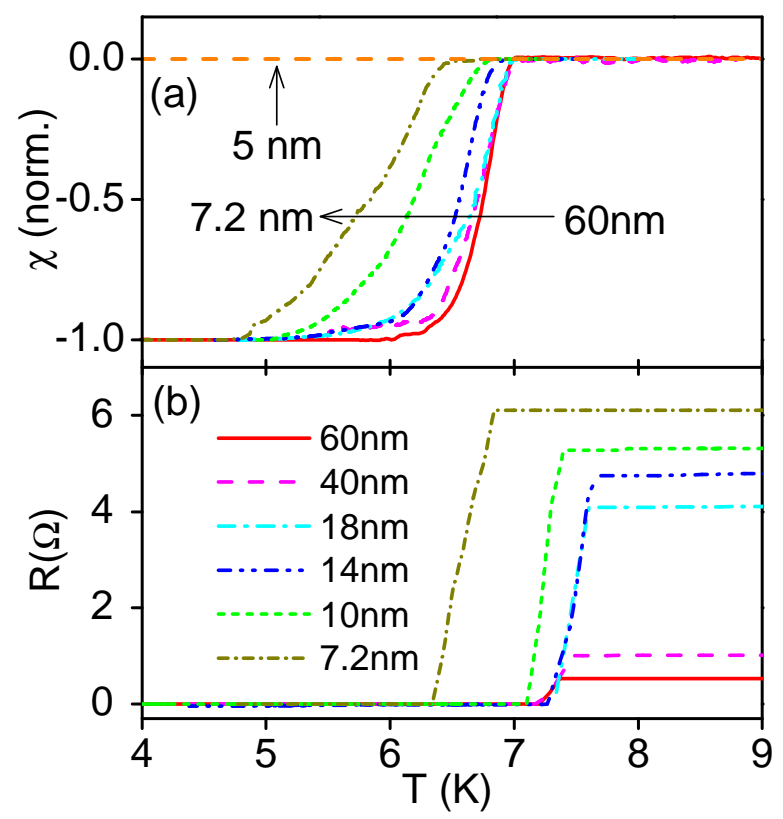

Figure 2 


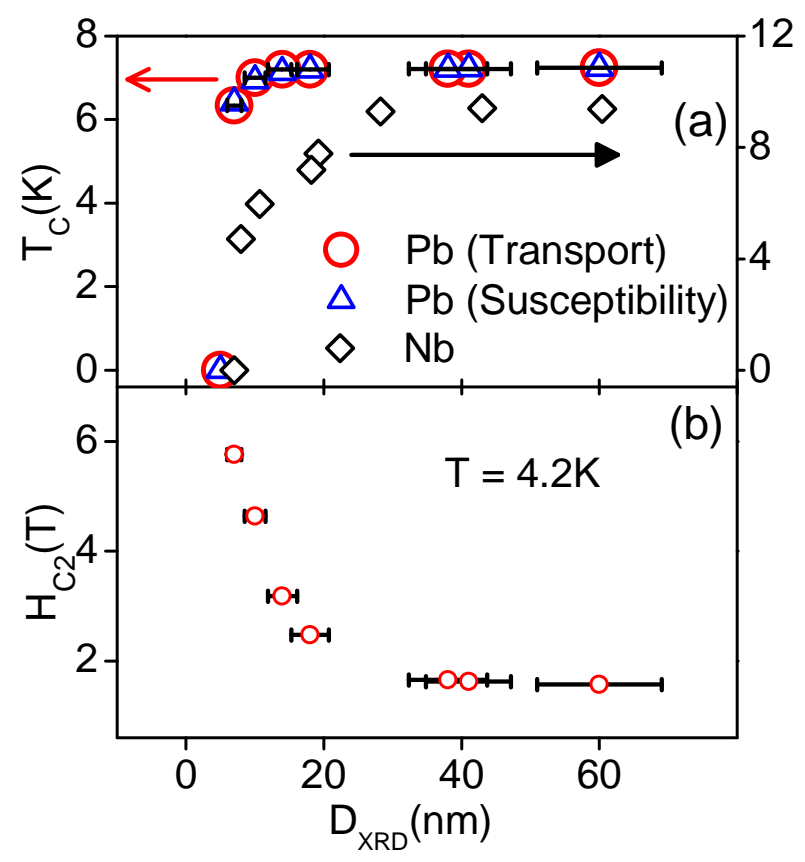

Figure 3 

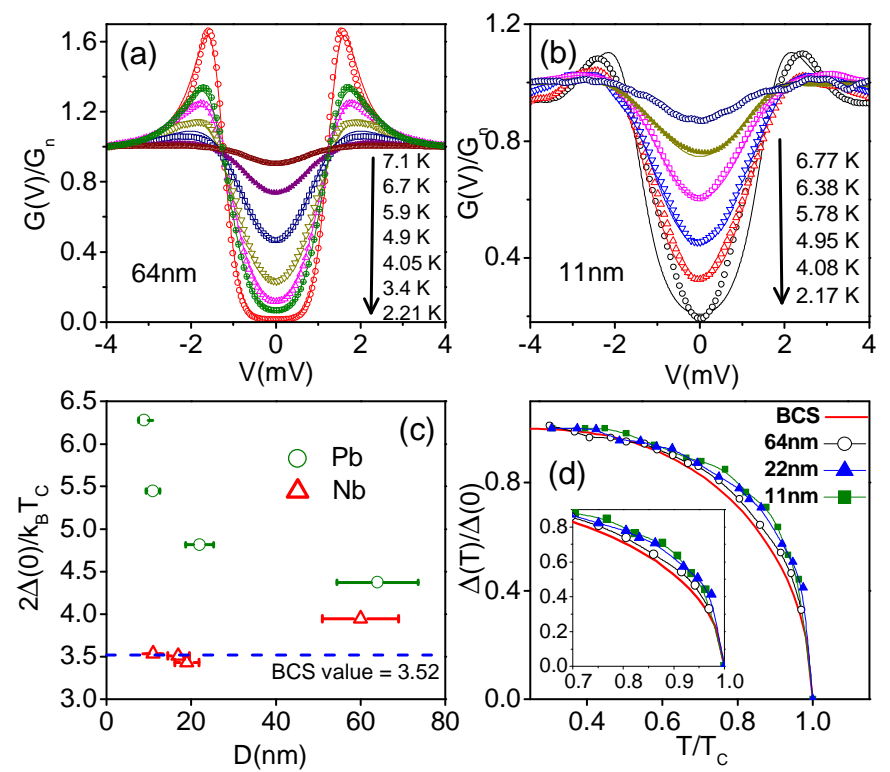

Figure 4 


\section{References}

1 S. Matsuo, H. Sugiura, and S. Noguchi, J. Low Temp. Phys. 15, 481 (1974).

2 P.W. Anderson, J. Phys. Chem. Solids 11, 26 (1959).

${ }^{3}$ K. Oshima, T. Kuroishi, T. Fujita, J. Phys. Soc. Jpn. 41, 1234 (1976).

4 B. Abeles, R. W. Cohen, G. W. Cullen, Phys. Rev. Lett. 17, 632 (1966).

5 N.A.H.K. Rao, J. C. Garland, D. B. Tanner, Phys. Rev. B 29, 1214 (1984); T. Tsuboi and T. Suzuki, J. Phys. Soc. Jpn. 42, 437 (1977).

${ }^{6}$ S. Reich, G. Leitus, R. Popovitz-Biro, M. Schechter, Phys. Rev. Lett. 91, 147001-1(2003).

7 W. H. Li, C.C.Yang, F. C. Tsao, K. C. Lee, Phys. Rev. B 68, 184507 (2003).

${ }^{8}$ H. M. Jaeger, D. B. Haviland, B. G. Orr, A. M. Goldman, Phys. Rev. B 40, 182 (1989), and references therein.

9 T. Hihara, Y. Yamada, M. Katoh, D. L. Peng, K. Sumiyama, J. Appl. Phys. 94, 7594 (2003).

10 R. P. Barber, Jr, L. M. Merchant, A. La Porta, R. C. Dynes, Phys. Rev. B 49, 3409 (1994); A. Frydman, O. Naaman, and R. C. Dynes, Phys. Rev. B 66, 052509 (2002)

11 D.C. Ralph, C.T. Black, and M. Tinkham, Phys. Rev. Lett. 74, 3241 (1995).

${ }^{12}$ W.-H. Li, C. C. Yang, F. C. Tsao, S. Y. Wu, P. J. Huang, M. K. Chung, and Y. D. Yao, Phys. Rev. B, 72, 214516 (2005)

${ }^{13}$ S. Bose, P. Raychaudhuri, R. Banerjee, P. Vasa, and P. Ayyub, Phys. Rev. Lett. 95, $147003(2005)$

14 J. M. Dickey, A. Paskin, Phys. Rev. Lett. 21, 1441 (1968).

15 W. L. McMillan, Phys. Rev. 167, 331 (1968). 
16 M. Strongin, R. S. Thompson, O. F. Kammerer, and J. E. Crow, Phys. Rev. B 1, 1078 (1970).

${ }^{17}$ M. Strongin, O. F. Kammerer, J. E. Crow, R. D. Parks, D. H. Douglass, M. A. Jensen, Phys. Rev. Lett. 21, 1320 (1968)

${ }^{18}$ Similar structure was also observed in nanocrystalline $\mathrm{Nb}$ films where electron energy loss spectroscopy confirmed the granular and intergranular region to be $\mathrm{Nb}$ and $\mathrm{Nb}_{2} \mathrm{O}_{5}$ respectively, see S. Bose, R. Banerjee, A, Genc, P. Raychaudhuri, H. L Fraser and P. Ayyub, J. Phys.: Condens. Matter 18, 4553 (2006).

${ }^{19}$ I. Giaever, Phys. Rev. Lett. 5, 147 (1960); I. Giaever, Phys. Rev. Lett. 5, 464 (1960)

${ }^{20}$ Sangita Bose, Pratap Raychaudhuri, Rajarshi Banerjee, and Pushan Ayyub, Phys. Rev. B 74, 224502 (2006).

${ }^{21}$ It has been shown in the context of anisotropic superconductors that the conductance spectrum arising from a distribution of superconducting energy gaps can be fitted by incorporating the broadening parameter $\Gamma$ : $\mathrm{P}$ Raychaudhuri, D Jaiswal-Nagar, Goutam Sheet, S Ramakrishnan and H Takeya, Phys. Rev. Lett. 93, 156802 (2004).

${ }^{22}$ R. C. Dynes, J. P. Garno, G. B. Hertel and T. P. Orlando, Phys. Rev. Lett. 53, 2437 (1984)

${ }^{23}$ Ivar Giaever and Karl Megerle, Phys. Rev. 122, 1101 (1961); P. Townsend and J. Sutton, Phys. Rev. 128, 591 (1962). 